\title{
L’ETP précédant la sortie du patient, une nouvelle frontière pour I'ETP
}

Reçu le 20 novembre 2017 / Accepté le 20 novembre 2017

La réduction des durées d'hospitalisation est devenue impérative pour les établissements de soins en tant que moyen de réduire les coûts de prise en charge des malades.

Cela concerne tous les secteurs d'hospitalisation: chirurgie (avec le développement de la chirurgie de jour), obstétrique, médecine, pédiatrie. Pour des raisons liées au souci d'efficacité dans le suivi du traitement, mais aussi pour des motifs d'ordre éthique, on considère aujourd'hui que le renvoi du patient à son domicile ne peut plus se concevoir sans une éducation.

Une abondante littérature anglo-saxonne (110 articles retrouvés sur Pubmed allant de 1999 à octobre 2017) est consacrée au concept et à la pratique de la «discharge education », littéralement : éducation proposée au patient avant sa sortie de l'hôpital que l'on peut tenter de raccourcir par l'acronyme: «ETP $s h$ »

Son efficacité a été démontrée dans des études randomisées contrôlées $(1,7)$ et des revues de la littérature $(9)$.

Grâce à une "discharge education » structurée, on a pu observer une amélioration des paramètres cliniques, allant jusqu'à une réduction de la mortalité $[1,2]$, une réduction des ré-hospitalisations précoces [3], une meilleure observance aux auto-soins [4], un gain de connaissances [5], une amélioration des paramètres psychologiques [6] et une plus grande satisfaction des patients $[7,8]$.

En France, cette pratique existe de façon plus ou moins formelle, se limitant souvent à des informations et conseils oraux, faisant moins fréquemment appel à des temps spécifiques d'éducation associés à une remise de recommandations écrites, plus rarement encore à des moyens numériques et/ou audio visuels d'illustration. Il n'y a pas, à notre connaissance, de pédagogie spécifique, ni de formation correspondante des soignants, ni même de recherche pédagogique sur ce format d'éducation thérapeutique. On retrouve là une situation à peu près similaire à celle de l'ETP avant les années quatre-vingt dix

Cette ETP $s h$ précédant la sortie du patient de l'hôpital est une action authentique d'éducation thérapeutique, même ciblée sur quelques compétences, même raccourcie dans le temps.

Comme tout autre format d'ETP, elle doit être structurée en reprenant les différentes étapes:

- repérage, par un diagnostic éducatif orienté sur le retour à domicile, des besoins essentiels du patient, en particulier sa capacité et/ou celle de son entourage à comprendre et appliquer les soins/ les conduites au domicile (hygiène, prise de médicaments, réfection de pansements, diététique, manipulation d'appareils, etc.);

- identification d'un nombre limité de compétences, mais assurant la sécurité du patient;

- échanges interactifs par l'usage de reformulations, utilisation d'outils pédagogiques adaptés : exercices de manipulation, remise de documents clairs et explicites, illustrations vidéo sur supports numériques: applications pour tablettes et smartphones, clés USB, sites web...

- planification d'un suivi pédagogique systématique (appels téléphoniques/ mails de rappel) favorisant une évaluation de l'application des compétences à domicile et leur réajustement;

- évaluation de l'efficacité de l'ETP $s h$, sur différents plans à déterminer (par exemple, clinique, cognitif, psychologique), tant au niveau de la personne soignée que du bilan de l'équipe.

L'ETP $s h$ est spécifique. Elle nécessite le recours à une pédagogie attentive d'autant qu'il existe des obstacles à son efficacité : le manque de compréhension de la part des patients de leur situation de santé, une mauvaise maîtrise de la langue, une faible littératie en santé [9], un état émotionnel qui nuit à l'attention. Cette action d'ETP concerne des situations pathologiques aiguës tout autant que chroniques:

- elle contribue à la sécurité du parcours de soin, à la gestion du risque clinique par la participation active du patient;

- elle est a priori individualisée, réalisée dans les heures qui précèdent la sortie du patient;

- elle concerne tout type de professionnel de santé formé à cette pratique;

- sa durée est 30 min à 1 heure, selon les cas, les objectifs, le contenu et/ou les capacités d'apprentissage du patient;

- elle comporte idéalement un suivi et peut nécessiter une reprise.

Sur le plan des références théoriques, elle fait appel aux travaux sur l'attention cognitive, à la gestion de l'émotion au cours de l'apprentissage, au transfert des acquis de l'éducation dans le contexte de vie du patient.

Aujourd'hui, le modèle «classique» de l'ETP, consacré aux maladies chroniques, fondé sur des programmes autorisés, 
s'adressant à des groupes de patients ne peut plus représenter le seul horizon de l'éducation thérapeutique. L'ETP précédant la sortie du patient, représente, par conséquent, un des futurs de l'ETP, tant sur le plan de la formation des éducateurs-soignants que sur celui de la recherche. Son format l'inscrit dans ce qui est appelé: les actions/activités d'éducation thérapeutique dont la reconnaissance par les autorités sanitaires permettrait d'étendre l'offre d'ETP.

Conflits d'intérêts. Les auteurs déclarent n'avoir aucun lien d'intérêt concernant les données publiées dans cet article.

\section{Références}

1. Koelling TM, et al. Discharge education improves clinical outcomes in patients with chronic heart failure. Circulation 2005; 111(2):179-185

2. Lord AS, et al. Discharge educational strategies for reduction of vascular events (DESERVE): design and methods. Int J Stroke 2015; 10 (A100):151-154

3. McLeod-Sordjan R, et al. Effectiveness of patient-caregiver dyad discharge interventions on hospital readmissions of elderly patients with community acquired pneumonia: a systematic review. JBI Libr Syst Rev 2011; 9(14):437
4. Kato N, et al. How effective is an in-hospital heart failure self-care program in a Japanese setting? Lessons from a randomized controlled pilot study. Patient Prefer Adherence 2016; 10:171-181.

5. Kommuri NVA, Johnson M, Koelling TM. Relationship between improvements in heart failure patient disease specific knowledge and clinical events as part of a randomized controlled trial. Patient Educ Couns 2012; 86(2):233-238.

6. Rushton $\mathrm{M}$, et al. Person-centred discharge education following coronary artery bypass graft: a critical review. J Clin Nurs 2017.

7. Majid N, Lee S, Plummer V. The effectiveness of orthopedic patient education in improving patient outcomes: a systematic review protocol. JBI database of systematic reviews and implementation reports $2015 ; 13(1): 122-133$.

8. Zhang C, et al. Clinical pharmacists on medical care of pediatric inpatients: a single-center randomized controlled trial. PloS One 2012; 7(1):e30856.

9. Polster D. Preventing readmissions with discharge education. Nurs Manag 2015; 46(10):30-37; quiz 37-38.

\section{Jean François d'Ivernois ${ }^{1, *}$, Rémi Gagnayre ${ }^{1}$ Maxime Morsa ${ }^{2}$}

${ }^{1}$ Laboratoire: Éducation et pratiques de santé (LEPS), EA 3412, Université Paris 13, Paris, France ${ }^{2}$ IPCEM, Paris, France ivernois.jf@orange.fr 